\title{
Antagonistic Effects of Rnd1 and RhoD GTPases Regulate Receptor Activity in Semaphorin 3A-Induced Cytoskeletal Collapse
}

\author{
Silvio M. Zanata, ${ }^{1,3}$ liris Hovatta, ${ }^{1}$ Beate Rohm, ${ }^{1}$ and Andreas W. Püschel ${ }^{1,2}$ \\ ${ }^{1}$ Molecular Neurogenetics Laboratory, Department of Neurochemistry, Max-Planck-Institute for Brain Research, D-60528 \\ Frankfurt, Germany, ${ }^{2}$ Institut für Allgemeine Zoologie und Genetik, Westfälische Wilhelms-Universität, D-48149 Münster, \\ Germany, and ${ }^{3}$ Ludwig Institute for Cancer Research, Cellular and Molecular Biology Laboratory, 01509-010 \\ Sao Paulo, Brazil
}

The semaphorins are a large protein family that is involved in the patterning of neuronal connections in the developing nervous system of both vertebrates and invertebrates. The chemorepulsive axon guidance signal Semaphorin 3A (Sema3A) induces the depolymerization of actin filaments and the collapse of sensory growth cones by activating a receptor complex that contains a plexin as the signal-transducing subunit. Here we show that, of a large number of GTPases tested, only Rnd1 and RhoD bind the cytoplasmic domain of Plexin-A1. Recruitment of active Rnd1 is sufficient to trigger signaling by

Semaphorin 3A (Sema3A) acts as a chemorepellent or chemoattractant on a wide variety of axons (Luo et al., 1993; Messersmith et al., 1995; Püschel et al., 1995; Kobayashi et al., 1997; Bagnard et al., 1998; Chedotal et al., 1998; Polleux et al., 1998, 2000). It induces a dramatic redistribution and depolymerization of actin filaments in sensory growth cones, ultimately resulting in their collapse (Fan et al., 1993; Fournier et al., 2000). Sema3A activates a receptor complex that contains Neuropilin-1 (Nrp-1) as the ligand binding subunit (He and Tessier-Lavigne, 1997; Kolodkin et al., 1997), a member of the plexin family as the signal transducing subunit (Takahashi et al., 1999; Tamagnone et al., 1999; Rohm et al., 2000a; Tamagnone and Comoglio, 2000), and the cell adhesion protein L1 (Castellani et al., 2000). Whereas Drosophila Plexin-A or human Plexin-B1 and Plexin-C1 can directly interact with Sema1a, Sema4B, and Sema7A, respectively, the class 3 semaphorins do not bind plexins but require a neuropilin as coreceptor (Comeau et al., 1998; Winberg et al., 1998; Takahashi et al., 1999; Tamagnone et al., 1999; Rohm et al., 2000a). Nrp-1 and Plexin-A1 are sufficient to reconstitute a functional Sema3A receptor in COS-7 cells. Similar to the induction of growth cone collapse by Sema3A, activation of the heterologously expressed receptor complex results in the collapse of COS-7 cells, presumably by inducing the depolymerization of actin filaments (Takahashi et al., 1999).

Received Aug. 17, 2001; revised Oct. 9, 2001; accepted Oct. 23, 2001.

This work was supported by the Deutsche Forschungsgemeinschaft (grants to A.W.P.) and the Deutscher Akademischer Austausch Dienst (fellowship to S.M.Z.). We thank B. Rahim and B. Kleiber for excellent technical assistance, S. Fuchs for help with sequencing, E. Evers and C. Klämbt for helpful discussions, and C. Burkhardt, G. Neufang, M. Bähler, and C. Klämbt for comments on this manuscript.

Correspondence should be addressed to Andreas W. Püschel, Institut für Allgemeine Zoologie und Genetik, Westfälische Wilhelms-Universität, Schloßplatz 5, D-48149 Münster, Germany. E-mail: apuschel@uni_muenster.de.

I. Hovatta's present address: The Salk Institute for Biological Studies, Laboratory of Genetics, 10010 North Torrey Pines Road, La Jolla, CA 92037.

Copyright (C) 2002 Society for Neuroscience $0270-6474 / 02 / 220471-07 \$ 15.00 / 0$
Plexin-A1, even in the absence of Sema3A, and initiates cytoskeletal collapse by activating its cytoplasmic domain. RhoD, in contrast, blocks Plexin-A1 activation by Rnd1 and repulsion of sympathetic axons by Sema3A. Thus, the antagonism of two GTPases regulates the activity of the Sema3A receptor, and activation by Rnd1 appears to be an essential step in signaling by Plexin-A1.

Key words: GTPases; cytoskeleton; neuropilin; plexin; actin; semaphorin

Members of the Rho family of small GTPases are central regulators of cytoskeletal dynamics and control the organization of actin filaments (Hall, 1998). The balance of Rho and Rac activity determines the cellular morphology of fibroblasts and neurons, and activation of Rho induces neurite retraction (Kozma et al., 1997; Sander et al., 1999; Luo, 2000; Wahl et al., 2000; Shamah et al., 2001). Dominant-negative RacN17 blocks Sema3A-induced growth cone collapse, which suggests that the Sema3A receptor regulates the activity of Rho-like GTPases (Jin and Strittmatter, 1997; Hall, 1998; Kuhn et al., 1999; Västrik et al., 1999). Most of the signaling events, however, that translate the binding of Sema3A to its receptor into structural changes of the cytoskeleton are unknown.

The cytoplasmic domain of plexins shows sequence similarity to Ras GTPase-activating proteins (GAPs) (Rohm et al., 2000b). This GAP homology includes two arginine residues that correspond to the invariant catalytic residues of Ras GAPs and are essential for the function of Plexin-A1 as Sema3A receptor, suggesting that plexins may act as GAPs to regulate the activity of small GTPases (Rohm et al., 2000b). Indeed, the Rho-like GTPases Rnd1 and Rac1 specifically interact with Plexin-A1 and Plexin-B1, respectively (Rohm et al., 2000b; Vikis et al., 2000; Driessens et al., 2001). The function of this interaction, however, remains unclear so far.

Here, we show that Plexin-A1 interacts not only with Rnd1 but also with RhoD. These GTPases have antagonistic effects on the activity of Plexin-A1. Whereas interaction of Rnd1 and Plexin-A1 triggers signaling by Plexin-A1 and results in cytoskeletal collapse, binding of RhoD has the opposite effect and blocks Plexin-A1 activity.

\section{MATERIALS AND METHODS}

Vectors. The expression vectors for alkaline phosphatase-tagged (AP) Sema3AP1b, hemagglutinin-tagged (HA) Nrp-1, vesicular stomatitis virus-tagged (VSV) Plexin-A1, glutathione $S$-transferase-tagged (GST) 


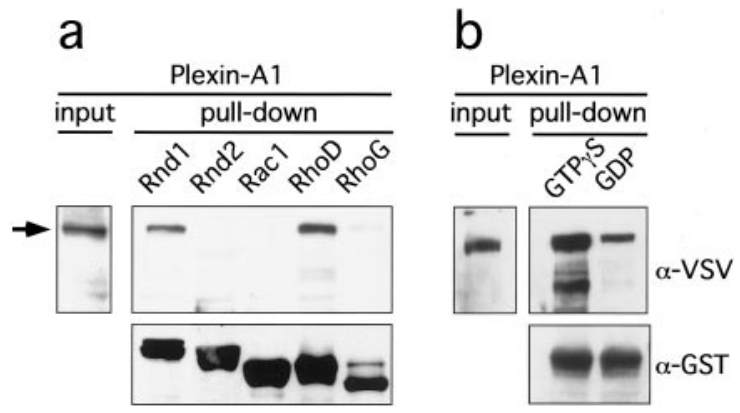

Figure 1. Interaction between Plexin-A1 and Rho-like GTPases. $a$, GST pull-down assays were performed with lysates of HEK 293T cells transfected with the expression vector pBK-VSV-Plexin-A1. Lysates were incubated with GST-Rnd1, -Rnd2, -Rac1, -RhoD, or -RhoG bound to glutathione-Sepharose beads and preloaded with GTP $\gamma \mathrm{S}$ as indicated. Bound proteins were analyzed by Western blot using an anti-VSV or anti-GST antibody. Plexin-A1 specifically bound to Rnd1 and RhoD. $b$, GST-RhoD was preincubated with GTP $\gamma \mathrm{S}$ or GDP as indicated and incubated with lysates of HEK 293T cells transfected with pBK-VSVPlexin-A1. Preincubation with GDP substantially reduced the amount of bound RhoD.

Rnd1, and GST-Rac1 have been described previously (Rohm et al., 2000a,b). Point mutations (Plexin-A1: P1, P1600G P2, LVG1598,1599,1600GGA; RhoD: G26V, T31N) were introduced into pBK-VSV-Plexin-A1 using the QuickChange mutagenesis kit (Stratagene, La Jolla, CA) according to the recommendations of the manufacturer. The coding sequences for Rnd2, RhoD, RhoG, R-Ras, RalA, N-Ras, M-Ras, RasD1, Rheb, Rit, Rin, Tcl, Rad, Rem, Rap1B, Arf6, and Rac3 were amplified from mouse embryonic day 12.5 (E12.5) spinal cord cDNA, and those for RhoH, Rif, Arh1, and Arh2 were amplified from human fetal brain cDNA (Clontech, Palo Alto, CA). PCR fragments were cloned into the pGEX4T2 (Amersham Biosciences, Freiburg, Germany) and the pEGFP-C1 vector (Clontech, Palo Alto, CA) and verified by sequencing. A oligonucleotide encoding the Src myristoylation signal (Buss et al., 1988) and a myc-tag was cloned into pBK-CMV and fused to the coding sequence of the cytoplasmic domain of Plexin-A1.

GST pull down. GST pull-down assays were done as described previously (Rohm et al., 2000a).

COS collapse assay. COS-7 cells were transfected using the Fugene 6 reagent (Roche, Mannheim, Germany). Two days after transfection, cells were incubated with AP-Sema3AP1b for $1 \mathrm{hr}$ at $37^{\circ} \mathrm{C}$ and fixed with $3.7 \%$ formaldehyde in PBS. AP-Sema3A and VSV-Plexin-A1 were detected with an anti-placental alkaline phosphatase (Chemicon, Temecula, CA) or an anti-VSV (Roche) antibody and Alexa Fluor 355- or Alexa Fluor 594-labeled secondary antibodies (Molecular Probes, Eugene, OR), respectively. The percentage of collapsed cells was determined according to the criteria of Takahashi et al. (1999). Examples of collapsed and noncollapsed cells are shown in Figure $2 a$. Occasionally $(<10 \%)$, partially collapsed cells were observed. These are included in the number of collapsed cells.

Coculture assay. Sympathetic ganglia were explanted from E9 chick embryos and transfected with the Helios Gene Gun System (Bio-Rad, München, Germany) as described previously (Rohm et al., 2000a). Transfected ganglia were cultured in a collagen matrix together with aggregates of HEK 293T cells transfected with the BK-SemaDP1b expression vector (Püschel et al., 1995; Adams et al., 1997) on poly-ornithine (Sigma, Taufkirchen, Germany)-coated coverslips for $24 \mathrm{hr}$, fixed with 4\% formaldehyde in PBS, and stained with phalloidin-rhodamine.

\section{RESULTS}

\section{Plexin-A1 interacts with Rnd1 and RhoD}

We showed previously that Plexin-A1 specifically interacts with Rnd1 but not RhoA or Rac1 (Rohm et al., 2000b). To identify additional GTPases that might bind Plexin-A1, we screened a collection of GST-GTPase fusion proteins by GST pull-down assays (Fig. 1 $a$, and data not shown). Of the 21 Ras- and Rho-like GTPases tested (see Materials and Methods), only RhoD showed a strong interaction with Plexin-A1. This interaction was GTP dependent because preloading of GST-RhoD with GDP resulted in a substantial reduction in the amount of Plexin-A1 recovered in a pull-down assay (Fig. 1b). The residual binding of Plexin-A1 probably results from an incomplete exchange of GDP for GTP by the bacterially expressed protein. However, we cannot rule out the possibility that GDP-RhoD binds to Plexin-A1 with a low affinity.

\section{RhoD blocks Sema3A-induced collapse}

To analyze the role of Rnd1 or RhoD for the function of Plexin-A1, we used a heterologous system that mimics growth cone collapse and has been used as a model to study the properties of semaphorin receptors (Takahashi et al., 1999; Rohm et al., 2000b). COS-7 cells become responsive to Sema3A upon coexpression of VSV-tagged Plexin-A1 and HA-tagged Nrp-1. The percentage of Plexin-A1-positive cells that collapsed after addition of Sema3A was determined by indirect immunofluorescence with an anti-VSV antibody (Fig. 2a). This modified protocol allowed to specifically analyze only those cells that coexpress the Sema3A receptor and an EGFP-GTPase fusion protein (Fig. 2b; see below). Addition of Sema3A to COS-7 cells coexpressing Nrp-1, Plexin-A1, and EGFP induced the collapse of $84.3 \pm 4.4 \%$ (mean $\pm \mathrm{SD}$ ) of Plexin-A1/EGFP double-positive cells (Fig. $2 a-c)$. Coexpression of either wild-type EGFP-RhoD or EGFPRhoDV26 with Plexin-A1 and Nrp-1 almost completely blocked Sema3A-induced collapse (Fig. 2b,c) and reduced it to levels (RhoDV26, $11.3 \pm 3.7 \%$; RhoD, $22.4 \pm 0.8 \%$ ) similar to those of untreated cells $(16.0 \pm 3.2 \%)$. RhoD appeared slightly less effective in blocking cell collapse than RhoDV26. In contrast, the dominant-negative mutant RhoDN31 had no effect (89.7 \pm $1.7 \%)$.

The heterologously expressed receptor may activate signal transduction pathways in COS-7 cells that differ from those responsible for the repulsion of axons. Therefore, we transfected sympathetic ganglia explanted from E9 chick embryos by particlemediated gene transfer with a mixture of tauEGFP and EGFP or EGFP-RhoDV26 and cultured them together with HEK 293T cells secreting Sema3A in a collagen gel matrix. Because of the strong repulsive effects of Sema3A, only a few short axons were detectable in the quadrant proximal to the aggregate after $24 \mathrm{hr}$ of culture when ganglia were transfected with a mixture of EGFP and tauEGFP (Fig. 3a,b,e). EGFP-positive cells tended to form short neurites, especially when located proximal to the source of Sema3A (Fig. 3a,b,e). When ganglia were transfected with expression vectors for EGFP-RhoDV26 and tauEGFP, however, most EGFP-positive neurons extended long axons, many of which were present in the proximal quadrant (Fig. $3 c, d, f$ ).

To quantify these effects, the ratio [proximal/distal (p/d) ratio] of axons that were not sensitive to the repulsive effects of Sema3A and extended into the proximal quadrant toward the Sema3Asecreting cell aggregate and those that extended in the opposite direction (Fig. 3e,f) was determined (Rohm et al., 2000a). In the control experiment (EGFP and tauEGFP), this ratio was $0.3 \pm$ $0.04(n=6)$ (Fig. $3 g$ ), reflecting an extension of axons predominantly into the distal direction. Expression of EGFP-RhoDV26 resulted in a p/d ratio of $1.24 \pm 0.16(n=6)$ because axons extended with equal probability toward and away from Sema3Aexpressing aggregates. Thus, RhoDV26 was able to block the effects of Sema3A not only in a heterologous system but also in Sema3A-sensitive neurons. 
a
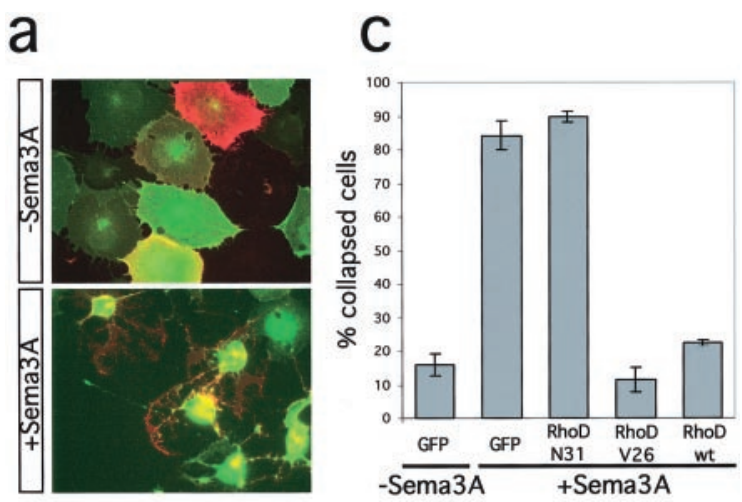

b

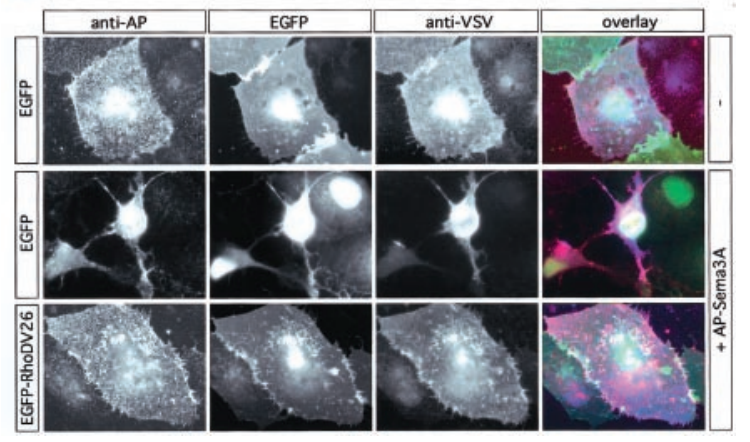

Figure 2. RhoD blocks Sema3A-induced collapse of COS-7 cells. a, COS-7 cells were transfected with the expression vectors pBK-VSVPlexinA1, pBK-HA-Nrp-1, and pEGFP and incubated with medium $(-\operatorname{Sema} 3 A)$ or with medium containing $0.4 \mathrm{~nm}$ AP-Sema3A $(+\operatorname{Sema} 3 A)$ for $1 \mathrm{hr}$ at $37^{\circ} \mathrm{C}$. Cells were fixed and processed for immunofluorescence without permeabilization. The expression of recombinant proteins was visualized by EGFP-fluorescence or indirect immunofluorescence using an anti-VSV antibody. $b$, COS-7 cells were transfected with the expression vectors pBK-VSV-PlexinA1, pBK-HA-Nrp-1, and pEGFP or pEGFPRhoDV26 as indicated and incubated with medium (-) or with medium containing $0.4 \mathrm{~nm}$ AP-Sema3A $(+A P$-Sema3A $)$ for $1 \mathrm{hr}$ at $37^{\circ} \mathrm{C}$. Bound AP-Sema3A was revealed using an anti-alkaline phosphatase antibody (red). The expression of recombinant proteins was visualized by EGFPfluorescence (green) or indirect immunofluorescence using an anti-VSV antibody (blue). c, The number of cells collapsed in response to Sema3A was determined according to published criteria (Takahashi et al., 1999). The percentage of collapsed cells ( gray bars) is displayed $(n=4 ; 200-300$ cells counted per experiment). wt, Wild type.

\section{Interaction of Rnd1 and Plexin-A1 induces collapse}

EGFP-Rnd1 had an effect opposite to that of RhoD when coexpressed with Plexin-A1 and Nrp-1 in COS-7 cells. Plexin-A1 and EGFP-Rnd1 double-positive cells displayed a collapsed morphology, even without addition of Sema3A. The presence of Rnd1 did not significantly increase the amount of cell collapse induced by Sema3A above the level seen with cells expressing only Plexin-A1 and Nrp-1 (data not shown). Coexpression of Plexin-A1 and Rnd1 resulted in the collapse of COS-7 cells also in the absence of Nrp-1 (66.7 $\pm 4.0 \%$ collapsed cells) (Fig. 4). Nrp-1 neither enhanced nor suppressed the collapsing effects of Rnd1 (data not shown). To test whether the cytoplasmic domain of Plexin-A1 was sufficient to cause cell collapse in association with Rnd1, we fused its coding sequence to a myristoylation signal (Buss et al., 1988) to generate myr-myc-PlexinA1. myr-myc-Plexin-A1 by itself did not induce cell collapse above background levels $(5.7 \pm 1.1 \%)$. Coexpression of myr-myc-Plexin-A1 and EGFP-Rnd1, however, was sufficient to collapse $49.9 \pm 4.7 \%$ of double-positive cells

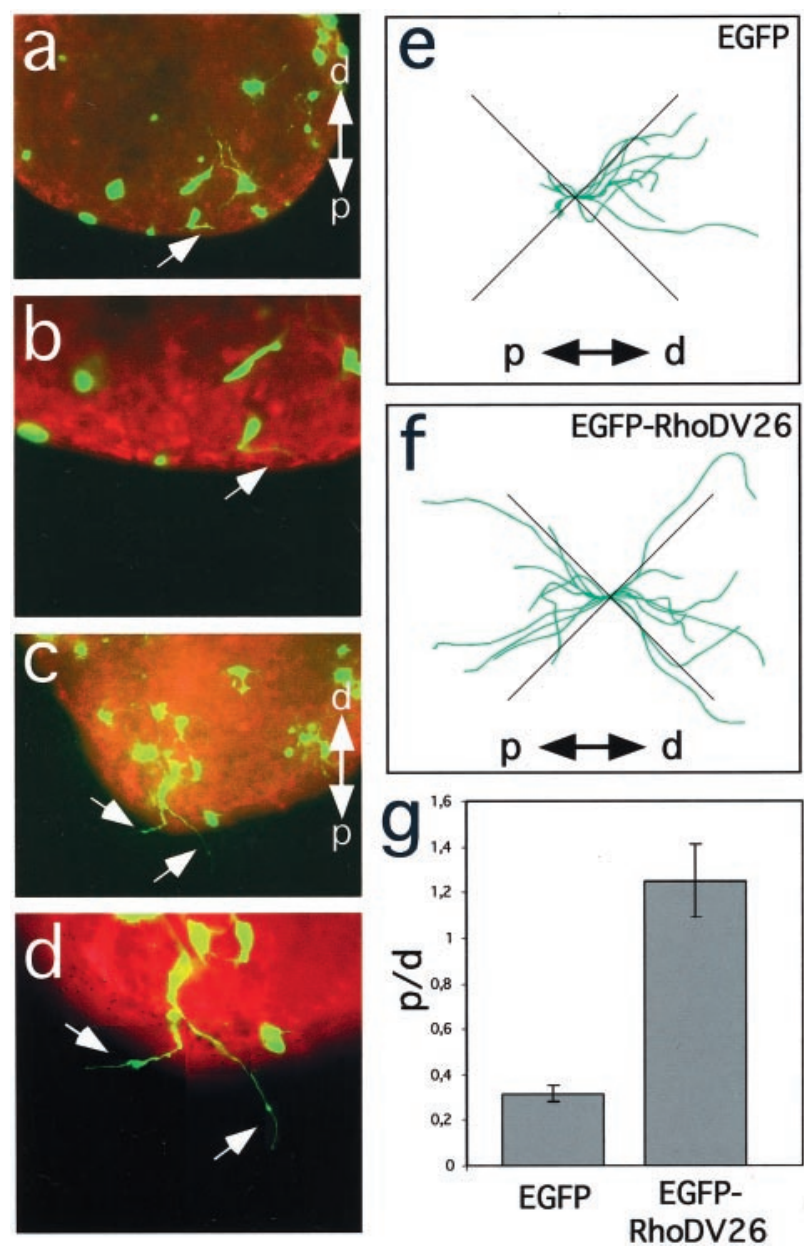

Figure 3. RhoD blocks repulsion of sympathetic axons by Sema3A. Explanted ganglia were transfected by particle-mediated gene transfer with a mixture of ptauEGFP and pEGFP $(a, b, e)$ or pBK-EGFPRhoDV26 $(c, d, f)$, cultured together with Sema3A-expressing cell aggregates and analyzed after $24 \mathrm{hr}$ of culture by following the trajectories of EGFP-positive axons (arrows). Cell aggregates are located outside the field of view (proximal is to the bottom). Staining with phalloidinrhodamine confirmed that no EGFP-negative axons were present in the proximal quadrant. Representative images of transfected ganglia are shown. $e, f$, A camera lucida representation of tauEGFP-positive axons extending into proximal $(p)$ and distal $(d)$ quadrants is shown. Axonal trajectories were recorded from neurons located at the proximal edge of explanted ganglia facing the Sema3A-secreting cell aggregate $(n=6)$. Axons extending into the lateral quadrants are not informative for their Sema3A sensitivity and were not included in our analysis. $g$, EGFPpositive axons extending toward $(p)$ or away $(d)$ from the cell aggregates secreting Sema3A were counted, and the ratio of axons growing proximally to that growing distally ( $\mathrm{p} / \mathrm{d}$ ratio) was calculated. Whereas axons extended preferentially away from Sema3A-expressing cells in control transfections, cells transfected with the expression vector for RhoDV26 had axons that extended with similar probability either proximally or distally ( $n=6,20-40$ ganglia per experiment).

(Fig. $4 a, b)$. Membrane tethering of the Plexin-A1 cytoplasmic domain was essential for this effect because deletion of the myristoylation signal abolished its collapsing activity $(9.6 \pm 3.1 \%$ collapse). Expression of Rnd1 alone did not result in cell collapse above background levels $(14.8 \pm 2.7 \%)$ (Fig. $4 b)$.

\section{RhoD blocks Rnd1-dependent collapse}

The ability of Rnd1 to induce the collapse of Plexin-A1expressing cells to an extent comparable with that seen after 

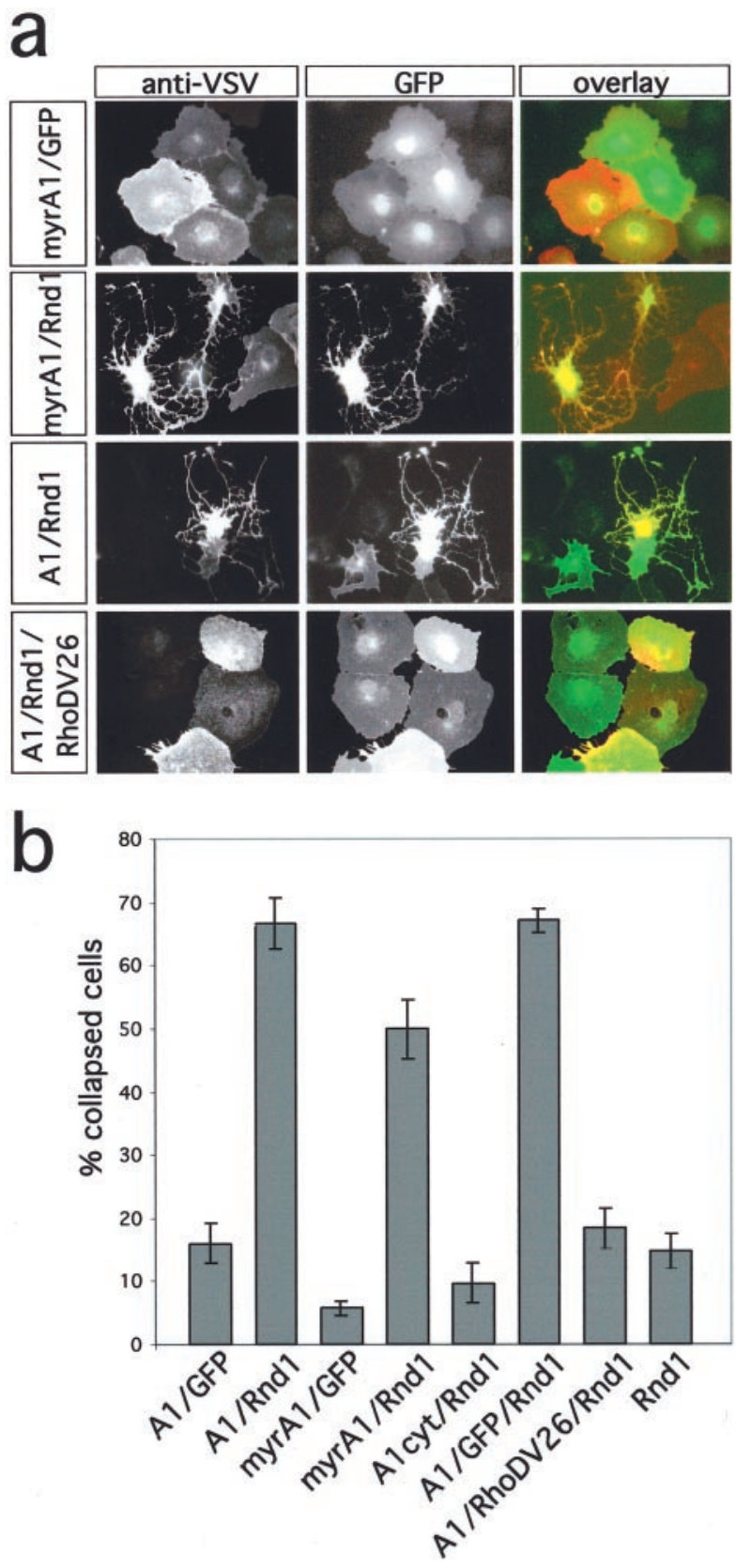

Figure 4. Interaction of Plexin-A1 and Rnd1 induces cell collapse. $a$, COS-7 cells were transfected with expression vectors for myr-mycPlexin-A1 (myrA1), VSV-Plexin-A1 (A1), and EGFP, EGFP-Rnd1 (Rnd1), or EGFP-Rnd1 and EGFP-RhoDV26 as indicated and processed for indirect immunofluorescence. The expression of recombinant proteins was visualized by EGFP-fluorescence (green) and immunofluorescence using an anti-myc or anti-VSV antibody (red). Interaction of the Plexin-A1 cytoplasmic domain and Rnd1 induces cell collapse. Rnd1-induced cell collapse is blocked by coexpression of RhoDV26. $b$, The number of cells collapsed in response to Sema3A was determined, and the percentage of collapsed cells ( gray bars) is displayed ( $n=4,200-300$ cells counted per experiment). Only membrane-bound Plexin-A1 was able to induce cell collapse during coexpression with Rnd1. Coexpression of the cytoplasmic Plexin-A1 domain without myristoylation signal (A1cyt) with Rnd1 was not able to induce cell collapse.

incubation with Sema3A suggests a role in the function of Plexin-A1 as a Sema3A receptor subunit. If Rnd1 is involved in signaling by Plexin-A1, this effect should be blocked by RhoD like the cytoskeletal collapse induced by Sema3A. Indeed, coexpres-
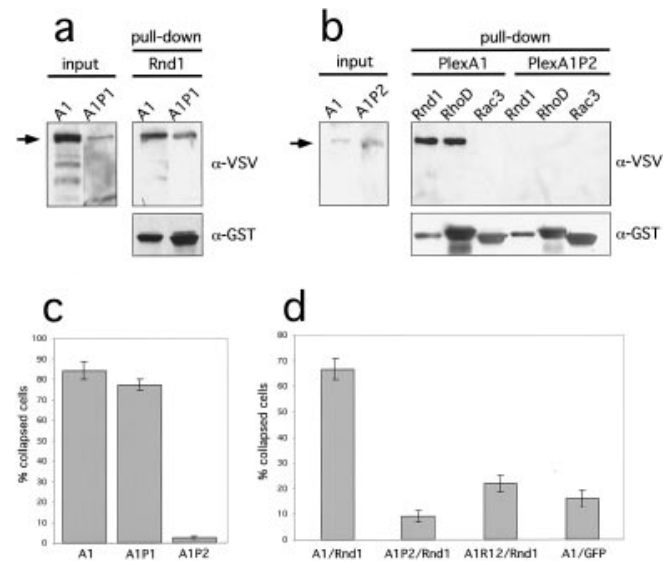

Figure 5. Mutations disrupting Rnd1 interaction or the GAP-homology region inactivate Plexin-A1. Lysates of HEK 293T cells transfected with expression vectors for VSV-Plexin-A1, VSV-Plexin-A1P1 $(a)$, or VSVPlexin-A1P2 $(b)$ were incubated with GST-Rnd1, -RhoD, or -Rac3 preloaded with GTP $\gamma \mathrm{S}$ as indicated, and bound proteins were analyzed by Western blot using an anti-VSV or anti-GST antibody. Plexin-A1P1 but not Plexin-A1P2 bound to Rnd1. $c$, COS-7 cells were transfected with expression for Nrp-1, EGFP, and VSV-PlexinA1, VSV-PlexinA1P1, or VSV-PlexinA1P2 and incubated with medium containing $0.4 \mathrm{~nm}$ APSema3A $(+\operatorname{Sema} 3 A)$ for $1 \mathrm{hr}$ at $37^{\circ} \mathrm{C}$. Cells were fixed and processed for immunofluorescence, and the number of cells collapsed in response to Sema3A was determined. The percentage of collapsed cells ( gray bars) is displayed ( $n=4,200-300$ cells counted per experiment). $d$, COS-7 cells were transfected with expression vectors for VSV-PlexinA1, VSVPlexinA1P2, VSV-PlexinA1R12, and EGFP-Rnd1 or EGFP as indicated and were processed for immunofluorescence, and the percentage of collapsed cells ( gray bars) was determined $(n=4,200-300$ cells counted per experiment). The mutants Plexin-A1P2 and Plexin-A1R12 were no longer able to induce cell collapse during coexpression with Rnd1.

sion of RhoDV26 with Plexin-A1 and Rnd1 blocked the collapse of COS-7 cells to a large extent $(18.3 \pm 3.2 \%$ collapsed cells compared with $67.0 \pm 1.8 \%$ after coexpression of Plexin-A1, EGFP, and EGFP-Rnd1) (Fig. 4b), supporting the idea that the recruitment of Rnd1 by Plexin-A1 plays an essential role in the function of Plexin-A1 that can be blocked by RhoD, as seen for the repulsion of sympathetic axons.

The Rho-related GTPase Rac1 was reported to bind to a CRIB-like sequence in Plexin-B1. A similar motif is also present in Plexin-A1 (Vikis et al., 2000; Driessens et al., 2001). We introduced two mutations into this sequence in Plexin-A1 and tested their effect on the interaction with Rnd1. Both mutants were transported to the cell surface with similar efficiency as Plexin-A1 (data not shown). Mutation of a single highly conserved proline residue (P1600G: Plexin-A1P1) had no effect on the binding of Rnd1 to Plexin-A1 in a GST pull-down assay (Fig. $5 a$ ). The corresponding mutation in Plexin-B1 also did not influence its interaction with Rac1 (B. Rohm and A.W. Püschel, unpublished observation). Mutation of three amino acid residues corresponding to that described by Vikis et al. (2000) for Plexin-B1 (LVP1598,1599,1600GGA: Plexin-A1P2), however, abolished binding of both Rnd1 and RhoD to the mutant Plexin-A1 protein (Fig. 5b). The ability of Plexin-A1 mutants to interact with GTPases correlated with their ability to mediate Sema3A-induced cell collapse after coexpression with Nrp-1 in COS-7 cells (Fig. 5c). Plexin-A1P1 but not Plexin-A1P2 was still able to induce cell collapse after addition of Sema3A. PlexinA1P2 also no longer induced cell collapse after coexpression with Rnd1 (Fig. 5d). 


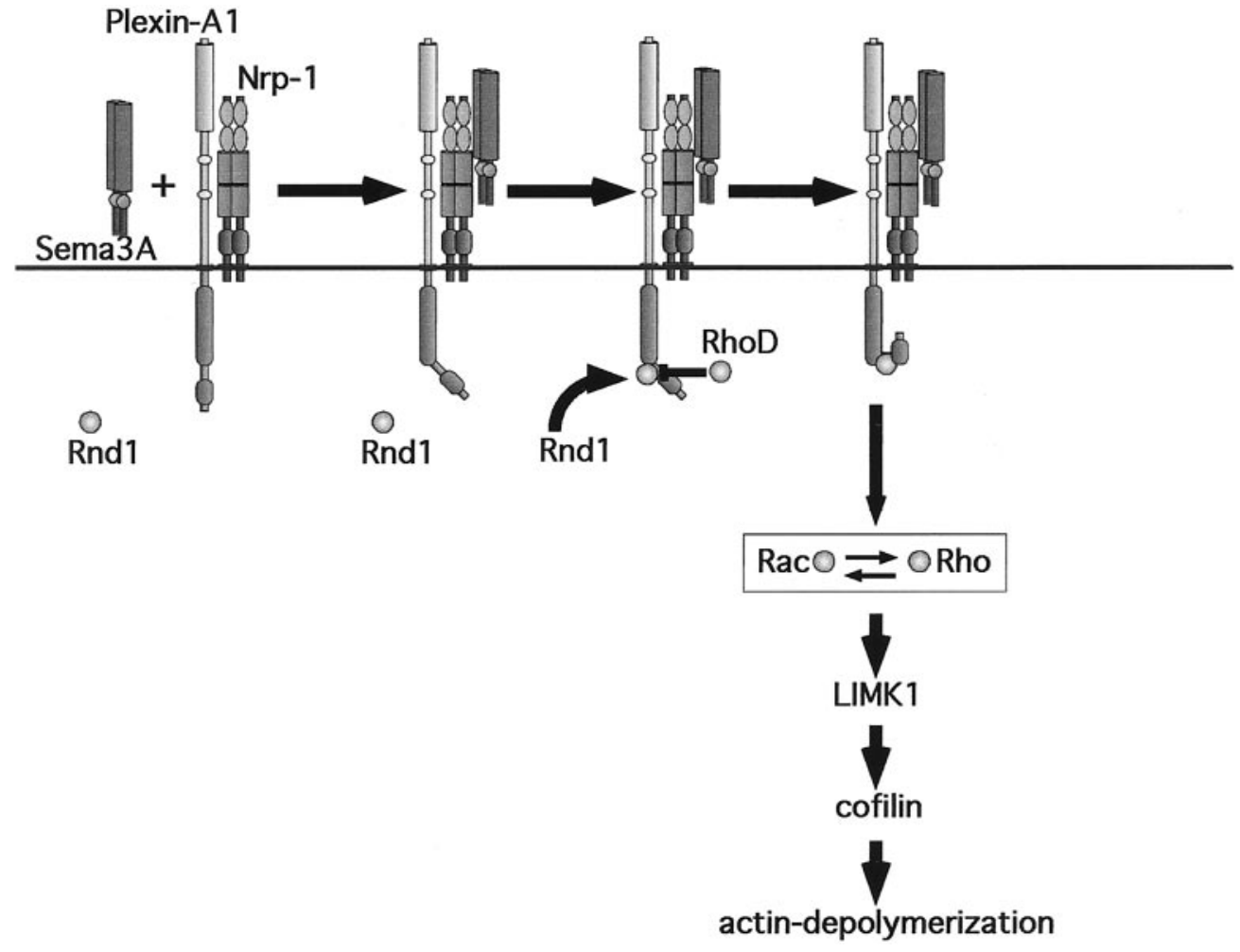

Figure 6. Plexin-A1, RhoD, and Rnd1 act like a logical AND gate. Sema3A binding to a receptor containing Nrp-1 as the ligand binding and Plexin-A1 as the signal transducing subunit triggers recruitment of Rnd1. Rnd1 has low intrinsic GTPase activity and is considered to be constitutively GTP bound. Interaction of Plexin-A1 and Rnd1 results in an activation of Plexin-A1 and downstream signaling events that shift the balance of Rac and Rho activity toward actin depolymerization. This process is blocked by interaction of Plexin-A1 with RhoD. The molecular components that link Rac and Rho to active Plexin-A1 are presently unknown.
To test whether membrane recruitment of Rnd1 is sufficient to induce cytoskeletal collapse, we used the Plexin-A1R12 construct that contains mutations in two conserved arginine residues within its GAP-homology domain and is incapable of signaling but still able to bind Rnd1 (Rohm et al., 2000b). When Plexin-A1R12 was coexpressed with EGFP-Rnd1, only $21.9 \pm 3.2 \%$ of doublepositive cells displayed a collapsed morphology. The requirement for an intact GAP-homology domain suggests that the primary function of the interaction with Rnd1 is the activation of signaling by the cytoplasmic domain of Plexin-A1 and not the recruitment of Rnd1 to the membrane.

\section{DISCUSSION}

\section{Rnd1 recruitment triggers signaling by Plexin-A1}

Here we show that Plexin-A1 interacts with two Rho-like GTPases, Rnd1 (54\% sequence similarity to RhoA; Nobes et al., 1998), and RhoD (59\% sequence similarity to RhoA; Murphy et al., 1996). Our data suggest that the recruitment of Rnd1 is an essential step in the induction of cytoskeletal collapse by PlexinA1. We propose that binding of Sema3A to the Nrp-1-Plexin-A1 receptor complex results in the recruitment of Rnd1, which activates Plexin-A1 and thereby initiates signaling by Plexin-A1 (Fig. 6). Rnd1, therefore, does not appear to have a role in the execution of cytoskeletal collapse downstream of Plexin-A1 but in its initiation by activating the cytoplasmic signaling domain of Plexin-A1. This conclusion is supported by three results. The cytoplasmic domain of Plexin-A1 is able to induce cell collapse during coexpression with Rnd1 in a heterologous system only when the GAP-homology domain is intact. A mutation in Plexin-A1 that abolishes its interaction with Rnd1 also blocks its ability to induce cell collapse. Finally, active RhoD binds to a site in Plexin-A1 that is identical to or overlapping with that for Rnd1 and blocks the collapse induced by Sema3A in a heterologous system, in sympathetic neurons, and during coexpression of Plexin-A1 and Rnd1.

The GAP-homology domain of Plexin-A1 is interrupted by an insert of 219 amino acid residues with no sequence similarity to other proteins (Maestrini et al., 1996; Rohm et al., 2000b; Driessens et al., 2001). This sequence is essential for the interaction with RhoD and Rnd1. Mutation of three residues within this sequence abolished the binding of Rnd1 and RhoD to Plexin-A1. Because both Rnd1 and RhoD use similar or identical binding sites, the block of collapsing activity by RhoD probably results from competition for the same binding motif. Alternatively, binding of RhoD could induce a conformational change in Plexin-A1 that blocks its interaction with Rnd1.

\section{The role of Rnd1 for Plexin-A1 signaling}

Rnd1 is thought to be a constitutively active GTPase, and its activity is likely to be determined by its subcellular localization (Nobes et al., 1998). Overexpression of Rnd1 probably saturates endogenous regulators, resulting in an excess of active Rnd 1 that induces cell collapse during binding to Plexin-A1. Mutation of conserved arginine residues within its GAP-homology domain is sufficient to completely inactivate Plexin-A1 without affecting its ability to interact with Rnd1 (Rohm et al., 2000b). Because this mutant cannot be activated by Rnd1, both Rnd1 recruitment and an intact GAP-homology domain are required for Plexin-A1 to initiate cell collapse. Based on these results, we suggest that interaction of Rnd1 with the insert sequence between the GAPhomology domains results in a conformational change that ultimately activates downstream signal transduction cascades, including Rac1, RhoA, LIM kinase 1, and cofilin that mediate growth cone collapse (Fig. 6) (Aizawa et al., 2001). The binding of Rac1 to the homologous sequence may have a similar function for Plexin-B1 as receptor for Sema4D (Tamagnone et al., 1999; Vikis 
et al., 2000). Our experiments do not resolve the question whether activation of Plexin-A1 is the sole function of Rnd1 or whether it also acts in conjunction with the GAP-homology domain to induce cell collapse. Rnd1 may also be a substrate for a possible GAP activity of Plexin-A1. However, so far, no GAP activity of Plexin-A1 for Rnd1 was detectable in vitro (Rohm and Püschel, unpublished results). Because an intact GAP-homology domain is essential for Plexin-A1-mediated collapse, we suggest that Rnd1 determines the activity of Plexin-A1 as a GAP that, in turn, regulates a yet to be identified GTPase. Alternatively, the sequence similarity of plexins and RasGAPs could indicate a structural rather then a functional conservation. Rnd1 binding might trigger the recruitment of adapter proteins to Plexin-A1 and thereby initiate cell collapse. Regardless of its biochemical activity, our results demonstrate that a functional cytoplasmic domain of Plexin-A1 is required for the induction of collapse by Rnd1.

\section{RhoD negatively regulates Sema3A signaling}

The ability of RhoD to suppress the activity of the Sema3Areceptor might reflect its involvement in a cross-regulation of semaphorin receptors by other signal transduction pathways. Increasing intracellular cGMP concentrations can switch repulsion by Sema3A to attraction (Song et al., 1998). It is conceivable that RhoD activity is regulated by a cGMP-responsive guanine nucleotide exchange factor similar to CNrasGEF (Pham et al., 2000). Increases in cGMP concentration would lead to suppression of Plexin-A1 activity by RhoD and could enable axons to display an attractive response to Sema3A. The axonal trajectories of RhoDV26-expressing sympathetic neurons, however, do not give an indication for attraction by Sema3A, which may require additional receptor or signaling components not present in sympathetic neurons. Sema3A stimulates endocytosis during growth cone collapse in addition to its effects on the cytoskeleton (Fournier et al., 2000). RhoD is present at the plasma membrane and on endosomal compartments and has been implicated in the regulation of endosomal dynamics (Murphy et al., 1996). An alternative possibility, therefore, is that RhoD terminates the activity of Sema3A receptors after their internalization to endosomes.

\section{Plexin-A1 signaling requires activation by Sema3A and Rnd1}

Our data suggest that Nrp-1, Plexin-A1, and two GTPases with antagonistic activities, Rnd1 and RhoD, are constituents of a complex molecular switch that induces the collapse of growth cones during activation by Sema3A. Within this complex, Plexin-A1 behaves like a logical AND gate upstream of Rac and Rho. Both Sema3A and Rnd1 have to activate Plexin-A1 to induce the collapse of cells or growth cones. The presence of active Rnd1 or RhoD, thus, determines the ability of Plexin-A1 to induce cell collapse during ligand binding. Intracellular signaling events coupled to active Plexin-A1, in turn, regulate the balance of Rac and Rho activity (Hall, 1998; Sander et al., 1999), which acts via LIM kinase 1 and cofilin to depolymerize actin filaments during growth cone turning and collapse.

\section{REFERENCES}

Adams RH, Lohrum M, Klostermann A, Betz H, Püschel AW (1997) The chemorepulsive activity of secreted semaphorins is regulated by furin-dependent proteolytic processing. EMBO J 16:6077-6086.

Aizawa H, Wakatsuki S, Ishii A, Moriyama K, Sasaki Y, Ohashi K, Sekine-Aizawa Y, Sehara-Fujisawa A, Mizuno K, Goshima Y, Yahara I (2001) Phosphorylation of cofilin by LIM-kinase is necessary for semaphorin 3A-induced growth cone collapse. Nat Neurosci 4:367-373. Bagnard D, Lohrum M, Uziel D, Püschel AW, Bolz J (1998) Semaphor- ins act as attractive and repulsive guidance signals during the development of cortical projections. Development 125:5043-5053.

Buss JE, Der CJ, Solski PA (1988) The six amino-terminal amino acids of p60src are sufficient to cause myristylation of p21v-ras. Mol Cell Biol 8:3960-3963

Castellani V, Chedotal A, Schachner M, Faivre-Sarrailh C, Rougon G (2000) Analysis of the L1-deficient mouse phenotype reveals cross-talk between Sema3A and L1 signaling pathways in axonal guidance. Neuron 27:237-249.

Chedotal A, JA DR, Ruiz M, He Z, Borrell V, De Castro F, Ezan F, CS G, Tessier-Lavigne M, Sotelo C, Soriano E (1998) Semaphorins III and IV repel hippocampal axons via two distinct receptors. Development 125:4313-4323.

Comeau MR, Johnson R, DuBose RF, Petersen M, Gearing P, VandenBos T, Park L, Farrah T, Buller RM, Cohen JI, Strockbine LD, Rauch C, Spriggs MK (1998) A poxvirus-encoded semaphorin induces cytokine production from monocytes and binds to a novel cellular semaphorin receptor, VESPR. Immunity 8:473-482.

Driessens MH, Hu H, Nobes CD, Self A, Jordens I, Goodman CS, Hall A (2001) Plexin-B semaphorin receptors interact directly with active Rac and regulate the actin cytoskeleton by activating Rho. Curr Biol 11:339-344.

Fan J, Mansfield SG, Redmond T, Gordon-Weeks PR, Raper JA (1993) The organization of F-actin and microtubules in growth cones exposed to a brain-derived collapsing factor. J Cell Biol 121:867-878.

Fournier AE, Nakamura F, Kawamoto S, Goshima Y, Kalb RG, Strittmatter SM (2000) Semaphorin3A enhances endocytosis at sites of receptor-F-actin colocalization during growth cone collapse. J Cell Biol 149:411-422.

Hall A (1998) Rho GTPases and the actin cytoskeleton. Science 279:509-514.

He Z, Tessier-Lavigne M (1997) Neuropilin is a receptor for the axonal chemorepellent Semaphorin III. Cell 90:739-751.

Jin Z, Strittmatter SM (1997) Rac1 mediates collapsin-1-induced growth cone collapse. J Neurosci 15:6256-6563.

Kobayashi H, Koppel AM, Luo Y, Raper JA (1997) A role for collapsin-1 in olfactory and cranial sensory axon guidance. J Neurosci 17:8339-8352.

Kolodkin AL, Levengood DV, Rowe EG, Tai YT, Giger RJ, Ginty DD (1997) Neuropilin is a semaphorin III receptor. Cell 90:753-762.

Kozma R, Sarner S, Ahmed S, Lim L (1997) Rho family GTPases and neuronal growth cone remodelling: relationship between increased complexity induced by $\mathrm{Cdc} 42 \mathrm{Hs}$, Rac1, and acetylcholine and collapse induced by RhoA and lysophosphatidic acid. Mol Cell Biol 17:1201-1211.

Kuhn TB, Brown MD, Wilcox CL, Raper JA, Bamburg JR (1999) Myelin and collapsin-1 induce motor neuron growth cone collapse through different pathways: inhibition of collapse by opposing mutants of rac1. J Neurosci 19:1965-1975.

Luo L (2000) Rho GTPases in neuronal morphogenesis. Nat Rev Neurosci 1:173-180.

Luo Y, Raible D, Raper JA (1993) Collapsin: a protein in brain that induces the collapse and paralysis of neuronal growth cones. Cell 75:217-227.

Maestrini E, Tamagnone L, Longati P, Cremona O, Gulisano M, Bione S, Tamanini F, Neel BG, Toniolo D, Comoglio PM (1996) A family of transmembrane proteins with homology to the MET-hepatocyte growth factor receptor. Proc Natl Acad Sci USA 93:674-678.

Messersmith EK, Leonardo ED, Shatz CJ, Tessier-Lavigne M, Goodman CS, Kolodkin AL (1995) Semaphorin III can function as a selective chemorepellent to pattern sensory projections in the spinal cord. Neuron 14:949-959.

Murphy C, Saffrich R, Grummt M, Gournier H, Rybin V, Rubino M, Auvinen P, Lutcke A, Parton RG, Zerial M (1996) Endosome dynamics regulated by a Rho protein. Nature 384:427-432.

Nobes CD, Lauritzen I, Mattei MG, Paris S, Hall A, Chardin P (1998) A new member of the Rho family, Rnd1, promotes disassembly of actin filament structures and loss of cell adhesion. J Cell Biol 141:187-197.

Pham N, Cheglakov I, Koch CA, de Hoog CL, Moran MF, Rotin D (2000) The guanine nucleotide exchange factor CNrasGEF activates ras in response to cAMP and cGMP. Curr Biol 10:555-558.

Polleux F, Giger RJ, Ginty DD, Kolodkin AL, Ghosh A (1998) Patterning of cortical efferent projections by semaphorin-neuropilin interactions. Science 282:1904-1906.

Polleux F, Morrow T, Ghosh A (2000) Semaphorin 3A is a chemoattractant for cortical apical dendrites. Nature 404:567-573.

Püschel AW, Adams RH, Betz H (1995) Murine semaphorin D/collapsin is a member of a diverse gene family and creates domains inhibitory for axonal extension. Neuron 14:941-948.

Rohm B, Ottemeyer A, Lohrum M, Püschel AW (2000a) Plexin/neuropilin complexes mediate repulsion by the axonal guidance signal semaphorin 3A. Mech Dev 93:95-104.

Rohm B, Rahim B, Kleiber B, Hovatta I, Püschel AW (2000b) The semaphorin $3 \mathrm{~A}$ receptor may directly regulate the activity of small GTPases. FEBS Lett 486:68-72. 
Sander EE, ten Klooster JP, van Delft S, van der Kammen RA, Collard JG (1999) Rac downregulates Rho activity: reciprocal balance between both GTPases determines cellular morphology and migratory behavior. J Cell Biol 147:1009-1022.

Shamah SM, Lin MZ, Goldberg JL, Estrach S, Sahin M, Hu L, Bazalakova M, Neve RL, Corfas G, Debant A, Greenberg ME (2001) EphA receptors regulate growth cone dynamics through the novel guanine nucleotide exchange factor ephexin. Cell 105:233-244.

Song H, Ming G, He Z, Lehmann M, McKerracher L, Tessier-Lavigne M, Poo M (1998) Conversion of neuronal growth cone responses from repulsion to attraction by cyclic nucleotides. Science 281:1515-1518.

Takahashi T, Fournier A, Nakamura F, Wang LH, Murakami Y, Kalb RG, Fujisawa H, Strittmatter SM (1999) Plexin-neuropilin-1 complexes form functional semaphorin-3A receptors. Cell 99:59-69.

Tamagnone L, Comoglio PM (2000) Signalling by semaphorin receptors: cell guidance and beyond. Trends Cell Biol 10:377-383.
Tamagnone L, Artigiani S, Chen H, He Z, Ming GI, Song H, Chedotal A, Winberg ML, Goodman CS, Poo M, Tessier-Lavigne M, Comoglio PM (1999) Plexins are a large family of receptors for transmembrane, secreted, and GPI-anchored semaphorins in vertebrates. Cell 99:71-80.

Västrik I, Eickholt BJ, Walsh FS, Ridley A, Doherty P (1999) Sema3Ainduced growth-cone collapse is mediated by Rac1 amino acids 17-32. Curr Biol 9:991-998.

Vikis HG, Li W, He Z, Guan KL (2000) The semaphorin receptor plexin-B1 specifically interacts with active rac in a ligand-dependent manner. Proc Natl Acad Sci USA 97:12457-12462.

Wahl S, Barth H, Ciossek T, Aktories K, Mueller BK (2000) Ephrin-A5 induces collapse of growth cones by activating Rho and Rho kinase. J Cell Biol 149:263-270.

Winberg ML, Noordermeer JN, Tamagnone L, Comoglio PM, Spriggs MK, Tessier-Lavigne M, Goodman CS (1998) Plexin A is a neuronal semaphorin receptor that controls axon guidance. Cell 95:903-916. 